\title{
The Role of Astronomy in Development: The Case of Uganda
}

\author{
Edward Jurua \\ Department of Physics, Mbarara University of Science and Technology, Uganda \\ email: ejurua@gmail.com
}

\begin{abstract}
Science and technology play a key role in economic development; and Universities have a direct stake in this process. A knowledge-based economy requires scientific and technological expertise that is strongly influenced by the strength of training in science and technology. However, in Uganda not many students opt for science subject at higher levels, and subsequently in the University. Therefore, there is need to encourage and motivate students to study science subjects in order for this to be successful. This can be achieved through introduction of stimulating subjects such as astronomy in the university curriculum. Astronomy is considered as the most appealing subject and an excellent tool for conveying scientific knowledge to young students. In this paper, the role that astronomy has played to motivate and interest students to study physics in Mbarara University of Science and Technology, in Uganda, is discussed.
\end{abstract}

Keywords. Astronomy, Ecomony, Development.

\section{Introduction}

The major economic activity in Uganda is Agriculture, and most of the Agricultural produce is exported in the raw form, thus generating low income for the government. As a result, the Government of Uganda embarked on the expansion of her industrial base and mechanization of Agriculture in order to generate more income. However, the current challenge is the unavailability of skilled, dedicated, and well-motivated human resource that is required to make such a programme successful. This resulted in government policies that aim to popularize and develop science and technology in order to provide the knowledge, skills, and professional and technical expertise that is required for the expansion of the industrial base and mechanization of Agriculture. However, very few students opt for science subjects and this has a direct effect on students admitted for sciences in tertiary institutions. Therefore, there is need to encourage and motivate students to study science subjects. This is discussed in the following section.

\section{Motivation of Students into Science}

To get the expertise in Science and Technology needed for economic development, young students should be motivated and attracted to the science subjects. Introducing subjects that motivate and attract young people into mathematics and science can do this. At this point, it is important to recognize the power of astronomy in attracting students into science subjects, which is vital for scientific and technological development. Fierro (1999) described Astronomy as the most appealing subject and an excellent tool for conveying scientific knowledge to young students. Besides, astronomical discoveries virtually interest everyone on the globe since astronomy is a frontier science (Anguma \& Jurua (2009)). Unlike other natural sciences, it takes the universe as its laboratory, in which physical laws and theories are applied, tested and refined at temperatures, pressures and scales unobtainable in terrestrial laboratories (Stobie (1995)). 
In order to promote science and technology in Uganda, Mbarara University of Science and Technology (MUST) introduced Astronomy as a course in the Physics department in 2003. The introduction of astronomy has not only created awareness among students, but also cultivated a lot of interest in pursuing further careers in astronomy. This has resulted in increased number of students opting for science, particularly in physics, in Mbarara University of Science and Technology. To make this a success, there is need to develop human resource in astronomy through postgraduate training. The plan for human resource development is discussed in the following section.

\section{Human Resource Development in Astronomy}

In 2012, the Department of Physics, MUST was invited to apply for the International Science Programme (ISP) support for human resource capacity development in astrophysics and space science. An application was submitted and the support was awarded for a period of three years (2014 - 2015); and this is renewed after every three years depending on the output of the project. The main aim is to develop human resource capacity in astronomy and space science. As a result of this project, the number of students that have cultivated interest in pursuing further careers in astronomy and space science in particular and physics in general has drastically increased. For instance, over 30 students (down from an average number of 8) applied for admission into Master of Science in Physics programme in Mbarara University of Science and Technology for the academic year $2015 / 2016$. This is an increase by more that $300 \%$.

During these first three years of ISP support, a number of students were supported directly and indirectly. In particular, 5 students graduated with Master of Science during the February 2015 graduation. Four (4) students are expected to complete their MSc and graduate during the next graduation in 2016. All these students have expressed interest to continue with $\mathrm{PhD}$ in astronomy. Two (2) students were recruited and supported for $\mathrm{PhD}$ programme; and they are expected to graduate during the next graduation ceremony in 2016. Many of our undergraduate students have also expressed interest to pursue further studies in Astronomy. However, there is still much more to be done facilitate the training in order to attain an optimum capacity.

\section{Conclusion}

The introduction of astronomy has resulted in increased number of students opting for science. However, there is still a critical need for continued support from both the government of Uganda and development partners in building research infrastructure and human resource capacity in Astronomy in Uganda. These will promote and generate new knowledge and continuously facilitate training in this discipline. This will in turn produce the skilled, dedicated, and well-motivated human resource required for technological development.

\section{References}

Anguma, K. S. \& Jurua, E. 2009 The Role of Astronomy in Society and Culture, Proceedings IAU Symposium, 260, 2009; D. Valls-Gabaud \& A. Boksenberg, eds.

Fierro, J. 1999, Teaching of Astronomy in Asia Pacific Region Bulletin, 15, 16

Stobie, R. S. 1995 Astrophysics and Space Science, 230, 9 